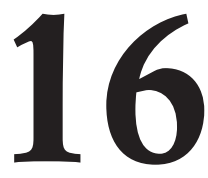

\title{
UNA INTERVENCIÓN PARA MEJORAR LA COMPETENCIA MEDIÁTICA E INFORMACIONAL
}

(AN INTERVENTION TO IMPROVE MEDIA AND INFORMATION LITERACY)

Sandra Liliana Cuervo-Sánchez

Juan Ignacio Martínez-de-Morentin

Concepción Medrano-Samaniego

Universidad del País Vasco (UPV/EHU)

DOI: $10.5944 / e d u c X X 1.30364$

Cómo referenciar este artículo/How to reference this article:

Cuervo-Sánchez, S.L., Martínez-de-Morentin, J.I. y Medrano-Samaniego, C. (2022). Una intervención para mejorar la competencia mediática e informacional. Educación XX1, 25(1), 407-431. https://doi.org/10.5944/educXX1.30364

Cuervo-Sánchez, S.L., Martínez-de-Morentin, J.I, \& Medrano-Samaniego, C. (2022). An intervention to improve media and information literacy. Educación XX1, 25(1), 407-431. https:// doi.org/10.5944/educXX1.30364

\section{RESUMEN}

El objetivo del presente artículo es aportar conocimiento a partir de los resultados de una intervención educativa para mejorar la Competencia Mediática e Informacional (CMI), realizada con 104 adolescentes de España (País Vasco) y Colombia (Bogotá), entre los 13 y 15 años de $3^{\circ}$ de ESO (Educación Secundaria Obligatoria). Previo a la intervención y para la evaluación pretest se aplicó el cuestionario CMI y, el mismo cuestionario, cambiando el contenido de algunos ítems, no la estructura, para los dos postest tras la intervención. La investigación se organizó en dos fases. La primera, la evaluación con pre-test y dos pos-test (inmediatamente después de la intervención y a los tres meses siguientes). La segunda, con la implementación de 11 sesiones en las cuales se desarrollaron las 
dimensiones de Acceso y uso de los medios de comunicación, de Lenguaje y comprensión crítica, de Procesos de producción y programación, y de Transformación de la realidad a través de la comunicación. El estudio, cuasiexperimental, se realizó con grupo experimental y control de ambos contextos y el diseño responde a un análisis de casos que se ha interpretado de forma contextualizada.

Para los análisis del impacto inmediato y diferido se utilizó un diseño factorial con covariante (ANCOVA). Entre los resultados destacan los cambios favorables en el contexto español en la dimensión de Lenguaje y comprensión crítica, en el impacto inmediato; y en el contexto colombiano en la dimensión de Procesos de Producción tanto en el impacto inmediato como diferido.

\section{PALABRAS CLAVE}

Competencia mediática, intervención, adolescencia, alfabetización mediática, enseñanza en medios

\section{ABSTRACT}

The aim of this paper is to generate knowledge based on the results of an educational intervention to improve Media and Information Literacy (MIL) carried out with 104 adolescents from Spain (Basque Country) and Colombia (Bogotá) aged between 13 and 15 years (year 3 of Compulsory Secondary Education). The CMI questionnaire was administered prior to the intervention for the pretest assessment. The same questionnaire was administered again after the intervention, at the two posttest times, with changes being made to the content of certain items, although not to the structure. The study was divided into two phases. The first comprised the pretest and two posttests (one immediately after the intervention and the second three months later). The second comprised 11 sessions focusing on four dimensions of MIL: Media access and use, Language and critical understanding, Production and programming processes, and Transformation of reality through communication. The study was quasi experimental and included an experimental and a control group in both contexts, following a case analysis design in which cases were interpreted in a contextualized manner.

To analyze the immediate and differed impacts, a covariate factorial design was used (ANCOVA). The most important results include positive changes in the Spanish context in the Language and critical understanding 
dimension immediately after the intervention; and positive changes in the Production processes dimension in the Colombian context, both immediately after the intervention and three months later.

\section{KEY WORDS}

Media competence, intervention, adolescence, media literacy, teaching in the media

\section{INTRODUCCIÓN}

En el contexto de pandemia a causa del COVID-19, la comunidad educativa se enfrentó a un intenso reto de digitalización y virtualización de las actividades escolares, a raíz de la manera imprevista en cómo se implementó el confinamiento global en marzo del 2020 (Garbe, Ogurlu, Logan y Cook, 2020). En medio de la crisis mundial, las cuestiones referidas a las competencias relacionadas con los medios de comunicación y la información tomaron un protagonismo en la vida del profesorado, el alumnado y las familias. Nuevamente emergieron discusiones sobre las brechas digitales a causa del acceso a los medios de comunicación, así como de los conocimientos necesarios para utilizar, comprender y producir conocimiento e información (Cabero y Valencia, 2021; Murillo y Duk, 2020). Igualmente, emerge la desinformación con un elemento a confrontar por parte de la ciudadanía y ser asumido desde los sistemas escolares (Elías y Catalan-Matamoros, 2020).

La Declaración de Seúl sobre la Alfabetización Mediática e Informacional para todos y por todos (UNESCO, 2020), agrupa las cuestiones históricas sobre la alfabetización mediática y el fenómeno actual de la desinformación. Dicha Declaración sugiere que en los medios de comunicación y en los proveedores de información las personas configuran sus percepciones, creencias y actitudes frente a la sociedad y el mundo que les rodea.

Hasta que este proceso se materialice, la educación escolar sigue estando interpelada como mediadora entre la sociedad, la industria mediática y los principios democráticos. Aportar contenidos y programas es, en la actualidad, una asignatura pendiente, dado el vacío curricular que existe frente a la Competencia Mediática e Informacional, a pesar de las múltiples recomendaciones que sobre el tema se han realizado a nivel mundial (Aguaded, Vizcaíno y Sandoval, 2019; Comisión Europea, 2006; Cuervo y Medrano, 2013). 
La Competencia Mediática e Informacional es un concepto que se estructura bajo el desarrollo de varios tipos de competencias y alfabetizaciones en los ámbitos de educación en medios de comunicación, información y nuevas tecnologías comunicativas (TICS) (Lau, 2013). En su definición se recogen habilidades, conocimientos y destrezas que tienen relación con la conciencia receptora y crítica frente a los medios de comunicación; así como con los mensajes, el manejo, comprensión y distribución de la información y el acceso y aprendizaje en el mundo digital (Lee, Lau, Carbo y Gendina, 2013). La UNESCO (2014) plantea una competencia en la cual incluye todos los tipos de medios de comunicación y proveedores de información, así como habilidades en tres niveles. El primero relacionado con las habilidades comunicativas de las personas; el segundo nivel, de recepción de la información, en el cual se incluye el acceso, la comprensión y la actitud crítica frente a los mensajes en los diferentes formatos mediáticos; y el tercer nivel relacionado con la divulgación y distribución de la información por parte de los ciudadanos.

Esta investigación se basó en un modelo de Competencia Mediática e Informacional, a partir de la definición propuesta por la UNESCO (2011) y Wilson, Grizzle, Tuazon, Akyempong y Cheung (2011), así como de las propuestas teóricas elaboradas, sobre competencia mediática, por Buckingham (2005), Ferrés y Piscitelli (2012), Mediasmarts (2014), Pérez Tornero y Varis (2012) y la Unión Europea (Directiva 2007/65/CE, artículo 37 y 2018/C 189/01).

Nuestro modelo plantea cuatro dimensiones, donde cada dimensión se estructura bajo categorías que sirven como indicadores de las habilidades, destrezas y conocimientos que las personas deberán tener sobre los medios de comunicación y la información (Cuervo, Foronda, Rodríguez, y Medrano, 2019).

La primera dimensión se denomina Acceso y uso de los medios de comunicación y está compuesta por tres categorías, Mecanismos de producción, Herramientas telemáticas y tecnologías y Uso del medio y la información. Se refiere a los hábitos de uso de los medios de comunicación, las habilidades metacognitivas para acceder a la información, así como los aspectos éticos y morales para la utilización de datos, aplicaciones e información (Pérez Tornero y Varis, 2012).

La segunda dimensión es Lenguaje y Comprensión Crítica, que la componen las categorías de Lectura y argumentación de imágenes, Creación a partir de imágenes y Criterios estéticos y juicios de valor. Está relacionada con los conocimientos y habilidades para identificar códigos, símbolos y estructuras que contienen los mensajes, así como la semántica, semiótica 
y las sintaxis implícitas en ellos. De igual manera, en esta dimensión se desarrollan las capacidades para reconocer las intencionalidades que existen en los procesos de comunicación (Buckingham, 2005).

La tercera dimensión se conoce como Procesos de producción y programación y las categorías que la componen son Fases de producción y Función cultural de los medios y la información. Esta dimensión se centra en los conocimientos y habilidades de los procesos de producción, programación, preproducción y postproducción de los medios de comunicación y la información. Es la dimensión que desarrolla los procesos más técnicos de la comunicación. De igual manera, plantea el desarrollo de una conciencia sobre la función social de los medios, tanto privados como públicos (Harshman, 2017) y los avances en la competencia digital del profesorado a partir de la Directiva de la Unión Europea en 2017 (Redecker, 2020) que facilitan la innovación en educación y disminuyen la brecha entre la cultura digital de los adolescentes y la manera tradicional de exponer contenidos en la escuela.

Finalmente, la cuarta dimensión se denomina Transformación de la realidad a través de la comunicación que la comprenden las categorías de Conciencia receptora y crítica, Competencia comunicativa y Apropiación tecnológica. Se relaciona con los conocimientos y actitudes frente a los medios de comunicación y la información en beneficio de los intereses comunes. En otras palabras, sería la participación ciudadana a través de los medios de comunicación. Está en consonancia con el uso que se hace de los medios para comprender el mundo, discernir discursos, textos, discusiones y debates, así como hacer frente a la industria de la comunicación (Hoechsmann, y Poyntz, 2012).

La educación en competencias aparece como parte de un proceso de modernización de los sistemas educativos, con una perspectiva funcionalista de la educación, que responde al proyecto económico y del conocimiento. Es un aprendizaje con un enfoque constructivista, centrado en el alumnado, donde el conocimiento se adquiere con la finalidad de resolver problemas y ser utilizado en la vida diaria. De este modo, el aprendizaje desarrolla capacidades, aptitudes, actitudes, destrezas y conocimientos (Villa y Poblete, 2007).

En Europa, este enfoque de competencias se estructura en la idea de competencias claves para vida. En España este enfoque se refleja en las diferentes leyes de educación, LOE (2006), LOMCE (2013) LOMLOE (2020) y con la adopción de las siguientes competencias básicas: Comunicación lingüística, Competencia matemática y competencias básicas en ciencia y tecnología, Competencia digital, Aprender a aprender, Competencias 
sociales y cívicas, Sentido de iniciativa y espíritu emprendedor y Conciencia y expresiones.

De acuerdo con Bernabéu, Esteban, Gallego, y Rosales (2011), en España, con la LOE (2006), la educación en medios es tratada desde diferentes perspectivas. En primer lugar, la educación con los medios, es decir, los medios como fuente de información. En segundo lugar, la educación en los medios, los medios como objeto de estudio, y tercer lugar, la educación ante los medios, en otras palabras, los medios como representación de la realidad. Situación que se mantiene en las siguientes leyes de educación.

En el caso colombiano, la educación también se basa en competencias claves, solo que en este contexto se reduce a cuatro competencias de orden académico y cívico que son: Competencias matemáticas, Competencias científicas (ciencias naturales y sociales), Competencias comunicativas y Competencias ciudadanas (MEN, 2006)

Desde ambas propuestas, y de acuerdo a las recomendaciones de la UNESCO, desde 1982, sobre la necesidad de educar en los medios de comunicación, ¿qué grado de Competencia Mediática e Informacional tienen los adolescentes? ¿es posible incrementar la Competencia Mediática e Informacional, en este ámbito, desde los programas de intervención? Es preciso destacar la complejidad comunicativa del proceso educativo dentro de la cultura digital, así como la convergencia de diferentes alfabetizaciones y competencias en la legislación educativa de ambos contextos culturales. En la figura 1 se sintetiza la confluencia de la alfabetización mediática, informacional y digital como paradigma de la educación en medios en la actualidad, tanto en España como en Colombia.

Revisado el estado del arte, este trabajo tiene como objetivo general analizar la competencia mediática e informacional, a través de la implementación de un Programa de Intervención con adolescentes, con objeto de favorecer el desarrollo de ciudadanos críticos y transformadores de su realidad comunicativa. 


\section{Figura 1}

Convergencia de alfabetizaciones en la cultura digital (Medrano, Cuervo, y Martínez de Morentin, 2019)

\begin{tabular}{|l|l|l|}
\hline \multicolumn{3}{|c|}{ Cultura digital } \\
\hline Alfabetización Mediática & Alfabetización Informacional & Alfabetización Digital \\
\hline \multicolumn{3}{|c|}{ Fundamentos } \\
\hline Estudios sobre medios & $\begin{array}{l}\text { Ciencias de la información y } \\
\text { proveedores }\end{array}$ & $\begin{array}{l}\text { Ciencia de la informática y la } \\
\text { computación }\end{array}$ \\
\hline \multicolumn{3}{|c|}{ Objetivos } \\
\hline $\begin{array}{l}\text { Comprender, evaluar y } \\
\text { utilizar los medios de } \\
\text { comunicación }\end{array}$ & $\begin{array}{l}\text { Acceso, evaluación y } \\
\text { distribución ética de la } \\
\text { información }\end{array}$ & $\begin{array}{l}\text { Capacidad de utilizar software e } \\
\text { infraestructuras digitales y } \\
\text { tecnologicas. } \\
\text { Conocimientos de gestión y } \\
\text { producción de contenidos } \\
\text { multimedia. }\end{array}$ \\
\hline $\begin{array}{l}\text { Promoción de los } \\
\text { derechos humanos } \\
\text { de la infancia y } \\
\text { libertad de expresión }\end{array}$ & $\begin{array}{l}\text { Desarrollo del } \\
\text { pensamiento critico y } \\
\text { reflexivo }\end{array}$ & $\begin{array}{l}\text { Desarrollo de } \\
\text { competencias de acceso, } \\
\text { evaluación, creación y } \\
\text { distribución }\end{array}$ \\
\hline
\end{tabular}

\section{METODOLOGÍA}

El diseño de esta investigación es un diseño pre-post con grupo de cuasi control. Estamos ante un diseño con intervención. Introduce grupos experimental y control, así como medidas pretest y postest. Este tipo de diseños se encuentran clasificados dentro de la categoría de cuasiexperimentales.

\section{Participantes}

Participaron 104 adolescentes de $3^{\circ}$ de la ESO (Educación Secundaria Obligatoria) en España (País Vasco) y Colombia (Bogotá). Las edades están comprendidas entre los 13 y los 15 años. El 43\% (N=45) fueron mujeres y $57 \%$ hombres $(\mathrm{N}=59)$. El grupo experimental del contexto español ha contado con el 30\% (N=31) de los participantes y en el contexto bogotano el $16 \%(\mathrm{~N}=17)$, mientras el grupo control, en el contexto español, fue de $33 \%$ $(\mathrm{N}=34)$ y en el bogotano de $21 \%(\mathrm{~N}=22)$. El alumnado participante en el estudio pertenecía a centros públicos y privados de educación formal. Las 
características socioeconómicas de los centros fueron similares, en ambos contextos. La muestra seleccionada es no probabilística e intencional.

\section{Instrumentos}

El instrumento de evaluación utilizado es un cuestionario de Competencia Mediática (CMI), validado en una prueba piloto y con evaluación de seis expertos. El cuestionario consta de 23 preguntas que analizan 11 categorías. Está conformado por diferentes escalas y evalúa los conocimientos de los sujetos como prueba de rendimiento. El carácter de dicho cuestionario es cuantitativo y cualitativo y no tiene una única escala Likert. Su fiabilidad estadística se calculó por dimensiones y por preguntas. Este cuestionario se cumplimentó antes de la intervención (T1pretest), inmediatamente después de terminar la intervención (T2-impacto inmediato), y tres meses después de terminar la intervención (T3-impacto diferido). Para llevar a cabo el proceso de intervención se desarrolló un conjunto de 11 sesiones didácticas que se implementaron en el aula, a lo largo de tres meses con una periodicidad semanal.

\section{Procedimiento}

El programa de intervención se adaptó a las características curriculares de cada centro educativo, en los grupos experimental y control. Para evitar el riesgo de contaminación del grupo experimental, se seleccionaron centros diferentes para los grupos control, conservando características socioeducativas similares entre los participantes.

Previo a la intervención se aplicó un cuestionario pretest en ambos grupos. El cuestionario utilizado, CMI, se ha basado en el cuestionario utilizado por Ferrés y Velilla (2011) tras una adaptación para esta investigación. La semana posterior, se inició el proceso de intervención en los dos grupos experimentales. Al finalizar las sesiones, una semana posterior, se aplicó un cuestionario postest (impacto inmediato) y a los tres meses otro cuestionario postest (impacto diferido) tanto en el grupo experimental como en el control. Los cuestionarios se cumplimentaron en una plataforma on-line, dentro del aula escolar.

La duración de la intervención fue de tres meses, con una periodicidad semanal por sesión. Se desarrollaron 9 unidades didácticas en 11 sesiones, cada una de 50 minutos. En la intervención se abordaron conceptos de las cuatro dimensiones de la Competencia Mediática e Informacional descrita en la introducción. Con una metodología basada en el pensamiento crítico 
y el aprendizaje significativo. En la figura 2 se puede observar la secuencia de las unidades didácticas realizadas en la Intervención.

Figura 2

Secuencia de las unidades didácticas de la Intervención

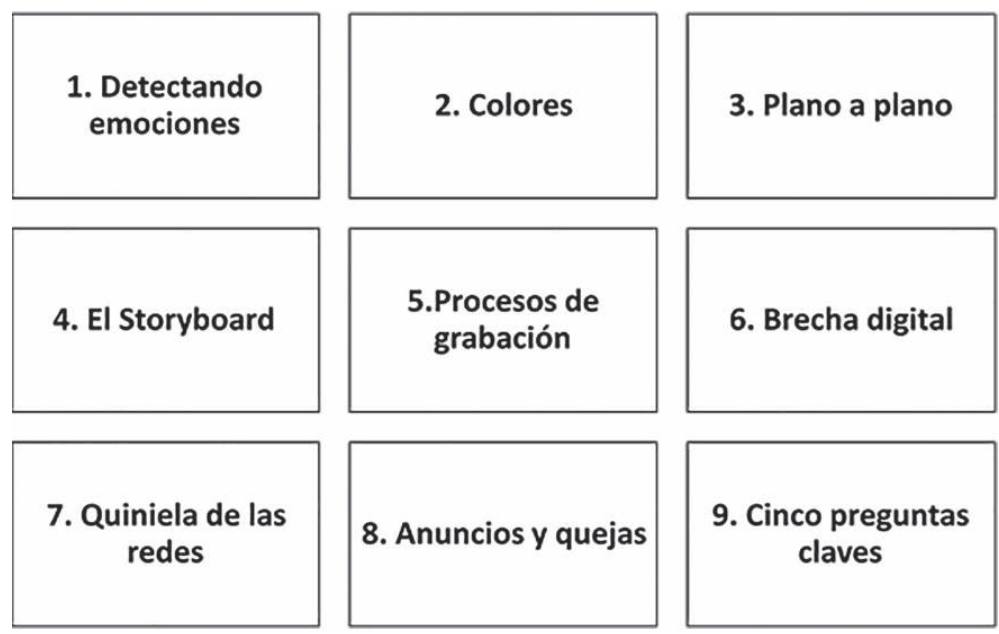

Esta investigación fue autorizada por el Comité de Ética de la Universidad del País Vasco y se ha requerido el consentimiento de cada participante y la autorización de los progenitores y la dirección de cada centro, previo a la aplicación del primer cuestionario pretest.

\section{Análisis de datos}

El análisis de datos se realizó con el paquete SPSS en su versión 20. Se elaboraron análisis descriptivos, tablas cruzadas y el análisis de varianza (ANCOVA) que tuvo como variable independiente el grupo (Experimental/ Control), como dependiente el resultado (post-test T2 para el impacto inmediato y post-test T3 para el impacto diferido) y como covariable el nivel de partida (pre-test T1). El coeficiente alpha de Cronbach se utilizó para el análisis de la fiabilidad de las escalas en los cuestionarios por dimensiones. En la dimensión de Acceso y uso de los medios de comunicación el alpha fue de .658; en la dimensión de Lenguaje y comprensión crítica fue de.130. El coeficiente bajo obtenido, muestra que cada una de las preguntas de esta dimensión miden competencias independientes y, por lo tanto, se han interpretado por separado en la evaluación del impacto de la intervención. Las preguntas 14, 16 y 17 son escalas de varios ítems por lo que su propósito es obtener una puntuación global en cada una de ellas. Es preciso analizar la consistencia interna de cada una de estas escalas. En las preguntas P14, 
P15 y P16 de la dimensión de Procesos de producción y programación el alpha de Cronbach fue de .381, .645 y .776 respectivamente. En la cuarta dimensión de Transformación de la realidad a través de la comunicación, el alpha de Cronbach se calculó por subescalas en la pregunta P.19 (sub-escala $1=.515$, sub-escala $2=.452$, sub-escala $3=.492$ ) y en la pregunta 22 (sub-escala $1=.298$, sub-escala $2=.655$, sub-escala $3=434$. sub-escala $4=.605$ ).

\section{RESULTADOS}

Los resultados obtenidos tras la intervención, en cada una de las dimensiones, en los contextos español y colombiano, se presentan por categorías con resultados estadísticamente significativos, tanto en el impacto inmediato como en el diferido. Se ha excluido el análisis de la dimensión de Acceso y uso de los medios de comunicación, ya que los resultados (T2 y T3) no fueron susceptibles de cambios en ninguno de los dos contextos.

La validez estadística como descripción de las categorías y las preguntas utilizadas se abordan ampliamente en Cuervo et al (2019). En las siguientes tablas se señalan las categorías evaluadas que presentan diferencias significativas y los resultados, que, no siendo significativos, presentan un $\eta^{2}$ mayor que 0.04 y con una media mayor en el grupo experimental (estos datos se resaltan en gris), dato razonable en el área de las ciencias sociales.

\section{Contexto español}

Dimensión de lenguaje y comprensión crítica

Tabla 1

Impacto inmediato y diferido en la Dimensión de lenguaje y comprensión crítica Grupos País Vasco

\begin{tabular}{lccccccc}
\hline \multicolumn{1}{c}{ Variables } & \multicolumn{3}{c}{ ANCOVA T2 } & \multicolumn{3}{c}{ ANCOVA T3 } \\
\hline $\begin{array}{l}\text { Dimensión Lenguaje-comprensión crí- } \\
\text { tica }\end{array}$ & F & Sig. & Eta $^{2}$ & F & Sig. & Eta $^{2}$ \\
\hline $\begin{array}{l}\text { Pr.6: Foto que mejor describe esta frase: } \\
\text { pensativa y triste, pero serena }\end{array}$ & .33 & .568 & .005 & .33 & .566 & .005 \\
\hline Pr.7: Por qué has elegido esta fotografía & 4.30 & .042 & .065 & 1.21 & .275 & .019 \\
\hline $\begin{array}{l}\text { Pr.8: Crea una historia coherente a partir } \\
\text { de un conjunto de imágenes }\end{array}$ & 5.63 & .021 & .083 & 4.43 & .039 & .067 \\
\hline $\begin{array}{l}\text { Pr.10: Las tres frases que mejor reflejan } \\
\text { los motivos estéticos por los cuales has } \\
\text { elegido el anuncio }\end{array}$ & 9.03 & .004 & .127 & .13 & .722 & .002 \\
\hline $\begin{array}{l}\text { Pr.12: Selecciona la frase con la que más } \\
\text { te identificas }\end{array}$ & 2.82 & .098 & .044 & 5.80 & .019 & .086 \\
\hline
\end{tabular}


Los resultados del impacto inmediato alcanzaron unos datos estadísticamente moderados como se observa en la Tabla 1. Los resultados por categorías plantean que la categoría de Criterios estéticos y juicios de valor - pregunta (Pr.10)_, la diferencia significativa es apreciable $(\mathrm{F}=9.03$, $\mathrm{p}<.05)$. Estos datos indican que, tras la intervención, en el primer postest (impacto inmediato) los adolescentes españoles tienen mayor facilidad para plantear criterios estéticos y, en menor medida, juicios de valor.

En la categoría de Creación a partir de imágenes, se obtiene una diferencia significativa apreciable de $\left(\mathrm{F}=5.63, \mathrm{p}<.05, \eta^{2}=.083\right)$ en la pregunta (Pr.8). Estos resultados pueden relacionarse con que la Intervención se desarrolló en la asignatura de Lengua, con mayor implicación del profesorado, relacionado con la disciplina y que las sesiones El Storyboard y Procesos de grabación lograron mayor impacto en el alumnado o generando una mayor motivación e implicación.

La categoría de Lectura y argumentación de imágenes alcanza resultados estadísticamente significativos en la pregunta (Pr.7) con una diferencia significativa de $\left(\mathrm{F}=4.30, \mathrm{p}<.05, \eta^{2}=.065\right)$. Lo que se entiende como una mejora en su habilidad argumentativa frente a la lectura de imágenes. Dichos resultados pueden asociarse con la motivación e implicación del alumnado y profesorado y con las sesiones de Detectando emociones y Colores.

En el impacto diferido, en esta dimensión, la categoría de Criterios estéticos y juicios de valor con la pregunta (Pr.12) conserva los resultados del impacto inmediato y es estadísticamente significativa con $(\mathrm{F}=5.80$, $\mathrm{p}<.05, \eta^{2}=.086$ ). El alumnado mantiene su habilidad para argumentar, ofrecer su opinión y juicios de valor frente a los productos audiovisuales y mediáticos a lo largo del tiempo. Podría plantearse como efectivo y con una interiorización a largo plazo de lo aprendido en las sesiones Detectando emociones y Colores.

La categoría de Creación a partir de imágenes, pregunta (Pr.8) es estadísticamente significativa con valores de $\left(\mathrm{F}=4.43, \mathrm{p}<.05, \eta^{2}=.067\right)$ y $(\mathrm{MC}=2.38)(\mathrm{ME}=1.42)$. Dicha paradoja puede relacionarse con un aspecto técnico a causa de la dificultad para cargar las fotografías en los ordenadores influenciando al alumnado del grupo experimental en dar respuestas al azar, y, por otro lado, en esta investigación se ha priorizado la validez ecológica, así ha podido ocurrir que el grupo control haya trabajado alguna actividad relacionada con la creación a partir de imágenes en alguna asignatura. 
Dimensión de procesos de producción y programación

Tabla 2

Impacto inmediato y diferido en la Dimensión de procesos de producción y programación - Grupos País Vasco

\begin{tabular}{lccccccc}
\hline \multicolumn{1}{c}{ Variables } & \multicolumn{3}{c}{ ANCOVA T2 } & \multicolumn{3}{c}{ ANCOVA T3 } \\
\hline \multicolumn{1}{c}{ Dimensión Procesos de Producción- } \\
$\begin{array}{l}\text { Programación } \\
\quad\end{array}$ & F & Sig. & Eta $^{2}$ & F & Sig. & Eta $^{2}$ \\
\hline $\begin{array}{l}\text { Pr.14: Unir las profesiones (audiovisua- } \\
\text { les) que aparecen en la columna de la iz- } \\
\text { quierda con las definiciones de la columna } \\
\text { derecha }\end{array}$ & 2.46 & .122 & .038 & 5.15 & .027 & .077 \\
\hline $\begin{array}{l}\text { Pr.15: Señalar a qué proceso de produc- } \\
\text { ción corresponde una secuencia, plan- } \\
\text { teada a través de un gráfico }\end{array}$ & 1.77 & .188 & .028 & .68 & .414 & .011 \\
\hline $\begin{array}{l}\text { Pr.16: Ordenar las acciones necesarias } \\
\text { para la realización de un audiovisual por } \\
\text { fases }\end{array}$ & 1.68 & .200 & .028 & .13 & .716 & .002 \\
\hline $\begin{array}{l}\text { Dimensión de Procesos de Producción- } \\
\text { Programación }\end{array}$ & 2.79 & .100 & .046 & 3.14 & .082 & .052 \\
\hline
\end{tabular}

En esta dimensión la comparación de medias de las diferentes preguntas no ofrece significatividad estadística, ni un tamaño del efecto apreciable. Estos resultados del impacto inmediato permiten inferir que las sesiones de Plano a plano, El Storyboard y Procesos de grabación no contribuyeron a mejorar la competencia mediática e informacional en este aspecto. Estas dimensiones sí contribuyeron en la Dimensión de Lenguaje y comprensión crítica.

Los resultados del impacto diferido, igual que en el impacto inmediato, no son estadísticamente significativos. Sin embargo, destacan las diferencias en la pregunta (Pr.14). Se puede intuir que el alumnado español incorpora, a largo plazo, las funciones relacionadas con el proceso de producción de un audiovisual. A pesar de ello, las fases y procesos de producción no son incorporados de la misma manera. En esta línea, las actividades desarrolladas en la Intervención no han sido suficientes para incorporar dicho saber, o al menos, con los datos disponibles no podemos afirmar que se han construido dichos aprendizajes. 
Dimensión de la transformación de la realidad a través de la comunicación

\section{Tabla 3}

Impacto inmediato y diferido Dimensión de la transformación de la realidad a través de la comunicación - Grupos País Vasco

\begin{tabular}{|c|c|c|c|c|c|c|}
\hline \multirow{2}{*}{$\begin{array}{c}\text { Variables } \\
\text { Dimensión Transformación realidad }\end{array}$} & \multicolumn{3}{|c|}{ ANCOVA T2 } & \multicolumn{3}{|c|}{ ANCOVA T3 } \\
\hline & $\mathbf{F}$ & Sig. & $\mathbf{E t a}^{2}$ & $\mathbf{F}$ & Sig. & $\mathbf{E t a}^{2}$ \\
\hline $\begin{array}{l}\text { Pr.3: Si el anuncio influye en la deci- } \\
\text { sión de compra de una persona ¿por qué } \\
\text { crees que lo logra? }\end{array}$ & 3.46 & .068 & .053 & 1.14 & .290 & .018 \\
\hline $\begin{array}{l}\text { Pr.4: Cuando las noticias van acompa- } \\
\text { ñadas de imágenes no hay tanto riesgo } \\
\text { de ser manipulado, porque gracias a las } \\
\text { imágenes se puede ver la realidad. }\end{array}$ & .16 & .692 & .003 & .46 & .499 & .007 \\
\hline $\begin{array}{l}\text { Pr.11: Si crees que el anuncio de Coro- } \\
\text { lla no se basa en la razón, ¿cómo piensas } \\
\text { que consigue que nos interesemos por el } \\
\text { coche? }\end{array}$ & .67 & .418 & .011 & .04 & .849 & .001 \\
\hline Pr.19.1: Programación & .03 & .864 & .000 & 1.37 & .247 & .022 \\
\hline Pr.19.2: Lenguaje & 12.86 & .001 & .174 & .25 & .617 & .004 \\
\hline Pr.19.3: Publicidad & .43 & .516 & .007 & 4.59 & .036 & .071 \\
\hline Pr.19.4: Derechos Humanos & 1.66 & .203 & .027 & .39 & .534 & .007 \\
\hline Pr.19.5: Información & .73 & .395 & .012 & .64 & .426 & .011 \\
\hline $\begin{array}{l}\text { Pr.19: ¿Hay razones para quejarse de } \\
\text { algunas televisiones? }\end{array}$ & 7.17 & .009 & .105 & .83 & .365 & .014 \\
\hline $\begin{array}{l}\text { Pr.21: La opción más apropiada para } \\
\text { discutir sobre lo visto en el último capí- } \\
\text { tulo de una serie favorita de televisión }\end{array}$ & 6.79 & .011 & .099 & 3.18 & .079 & .050 \\
\hline Pr23.1: Trabajo colectivo & .45 & .508 & .008 & 3.43 & .069 & .059 \\
\hline Pr23.3: Participación ciudadana & .01 & .932 & .000 & 1.26 & .266 & .022 \\
\hline Pr23.4: Debate & 4.41 & .041 & .078 & 4.76 & .034 & .084 \\
\hline Pr23.5: Comentario y/o cotilleo & 6.97 & .011 & .107 & .07 & .797 & .001 \\
\hline
\end{tabular}

En el impacto inmediato la dimensión obtiene resultados estadísticamente significativos, en varias preguntas, en las categorías que conforman ésta, como se aprecia en la Tabla 3. En la categoría de Conciencia receptora y crítica, se puede plantear que el alumnado, al terminar la intervención, es más propenso a quejarse por el lenguaje utilizado en los medios de comunicación. Podría decirse que las sesiones Anuncios y Quejas y Cinco preguntas claves, que abordan el análisis, la comprensión y 
la evaluación, tanto de los mensajes como de los medios, redundó a corto plazo en el alumnado español.

En Conciencia receptora y crítica, los resultados del impacto inmediato no son estadísticamente significativos. El alumnado necesita trabajar su capacidad de reflexión y análisis crítico de la realidad comunicativa de su entorno. Las sesiones relacionadas con procesos de metacognición, deben realizarse en un proceso más largo y cuidadoso ya que es conveniente profundizar estos procesos a partir de estrategias visuales como las utilizadas en la dimensión de Lenguaje y comprensión crítica.

En la Competencia comunicativa los resultados del impacto inmediato han sido estadísticamente significativos, con una diferencia significativa de $(\mathrm{F}=6.79, \mathrm{p}<.05)$ en la pregunta (Pr.19). Dichos resultados podrían relacionarse con su realidad comunicativa inmediata, en la cual la vida off-line se mantiene conectada con la realidad on-line. A pesar de ello, se observa que, en los datos de la categoría de Conciencia receptora y crítica, la utilización de la tecnología no se corresponde con una mayor capacidad crítica o una mayor capacidad creativa con los medios de comunicación.

En la categoría de apropiación tecnológica los resultados del impacto inmediato, son estadísticamente significativos en los valores de dos de los ítems (Pr.23.4) con una significación de $\left(\mathrm{F}=4.41, \mathrm{p}<.05, \eta^{2}=.078\right)$. Así como en el ítem (Pr.23.5) con un $\left(\mathrm{F}=6.97, \mathrm{p}<.05, \eta^{2}=.107\right)$. Es posible plantear que los adolescentes de este contexto tienen mayor facilidad para apropiarse de los medios de comunicación, sobre todo, en actividades en las que mantienen relaciones sociales con sus compañeros y tienen dificultades en otros escenarios de participación en lo público con las mismas habilidades.

En relación con el impacto diferido se mantienen resultados estadísticamente significativos en la categoría de apropiación tecnológica, En el ítem debate (Pr.23.4) la significación fue de ( $\left.\mathrm{F}=4.76, \mathrm{p}<.05, \eta^{2}=.084\right)$ Podría decirse que el alumnado mantiene a lo largo del tiempo los conocimientos relacionados con la utilización de los medios de comunicación acorde con la finalidad del mensaje. 
Contexto de Bogotá (Colombia)

Dimensión de lenguaje y comprensión crítica

\section{Tabla 4}

Impacto inmediato y diferido en la Dimensión de lenguaje y comprensión críticaBogotá

\begin{tabular}{lccccccc}
\hline \multicolumn{1}{c}{ Variables } & \multicolumn{3}{c}{ ANCOVA T2 } & \multicolumn{4}{c}{ ANCOVA T3 } \\
\hline Dimensión Lenguaje-comprensión crítica & F & Sig. & Eta $^{2}$ & F & Sig. & Eta $^{2}$ \\
\hline $\begin{array}{l}\text { Pr.6: Foto que mejor describe esta frase: } \\
\text { pensativa y triste, pero serena }\end{array}$ & 1.54 & .222 & .041 & 4.73 & .036 & .116 \\
\hline Pr.7: Por qué has elegido esta fotografía & .22 & .639 & .006 & .60 & .443 & .016 \\
\hline $\begin{array}{l}\text { Pr.8: Crea una historia coherente a partir } \\
\text { de un conjunto de imágenes }\end{array}$ & .50 & .484 & .014 & 4.41 & .043 & .109 \\
\hline $\begin{array}{l}\text { Pr.10: Las tres frases que mejor reflejan } \\
\text { los motivos estéticos por los cuales has } \\
\text { elegido el anuncio }\end{array}$ & 3.70 & .062 & .093 & .98 & .329 & .026 \\
\hline $\begin{array}{l}\text { Pr.12: Selecciona la frase con la que más } \\
\text { te identificas }\end{array}$ & .08 & .779 & .002 & 1.15 & .290 & .031 \\
\hline
\end{tabular}

Los resultados del impacto inmediato, en todas las categorías de esta dimensión son empíricamente moderados. Las diferencias no son significativas. A pesar de ello, en dos categorías, se encuentran diferencias relevantes según el indicador del tamaño del efecto (Tabla 4). Es decir, teniendo en cuenta el $\left(\eta^{2}\right)$ sí se pueden observar diferencias en las medias entre el grupo experimental y el grupo control, antes y después del proceso de intervención.

Los resultados podrían relacionarse con el nivel de asimilación de algunas de las actividades de las sesiones de intervención. Por ejemplo, en las sesiones de Detectando emociones y Colores que abordan las categorías de Criterios estéticos y juicios de valor y refuerzan la categoría de Lectura y argumentación de imágenes. Estas sesiones se centran en las emociones y sentimientos en la adolescencia. Es un tema primordial que pudo incidir en la motivación para el aprendizaje, mucho más que otras sesiones más conceptuales.

En los resultados de esta dimensión hay que destacar que en las preguntas que exigen una mayor elaboración las puntuaciones son más bajas, lo que podría interpretarse como una mayor dificultad para el desarrollo del pensamiento abstracto en relación con la reflexión sobre emociones y sentimientos. 
En los resultados del impacto diferido, la categoría de Creación a partir de imágenes obtuvo un resultado estadísticamente significativo y con un tamaño del efecto apreciable $\left(\mathrm{F}=4.41, \mathrm{p}<.05, \eta^{2}=.109\right)$ en la pregunta (Pr.8). Se puede interpretar que el alumnado del grupo experimental ha consolidado el aprendizaje en cuanto a la creación a través de imágenes de las sesiones denominadas El storyboard y Procesos de grabación.

En la categoría Lectura y argumentación de imágenes y de Criterios estéticos y juicios de valor, los resultados son paradójicos en el impacto diferido, el grupo control alcanzó una media mayor que del grupo experimental en la pregunta (Pr.6) ( $\mathrm{MC}=2.58$ y $\mathrm{ME}=1.36)$. Como se ha citado con anterioridad el grupo control pudo recibir un refuerzo en esta temática.

Dimensión de procesos de producción y programación

Tabla 5

Impacto inmediato y diferido en la Dimensión de procesos de producción y programación - Grupos Bogotá

\begin{tabular}{|c|c|c|c|c|c|c|}
\hline \multirow{2}{*}{$\begin{array}{c}\text { Variables } \\
\text { Dimensión Procesos de Producción- } \\
\text { Programación }\end{array}$} & \multicolumn{3}{|c|}{ ANCOVA T2 } & \multicolumn{3}{|c|}{ ANCOVA T3 } \\
\hline & $F$ & Sig. & $\mathrm{Eta}^{2}$ & $\mathrm{~F}$ & Sig. & $\mathrm{Eta}^{2}$ \\
\hline $\begin{array}{l}\text { Pr.14: Unir las profesiones (audiovi- } \\
\text { suales) que aparecen en la columna de } \\
\text { la izquierda con las definiciones de la } \\
\text { columna derecha }\end{array}$ & 9.89 & .003 & .216 & 8.94 & .005 & .199 \\
\hline $\begin{array}{l}\text { Pr.15: Señalar a qué proceso de pro- } \\
\text { ducción corresponde una secuencia, } \\
\text { planteada a través de un gráfico }\end{array}$ & 10.49 & .003 & .226 & 2.75 & .106 & .071 \\
\hline $\begin{array}{l}\text { Pr.16: Ordenar las acciones necesarias } \\
\text { para la realización de un audiovisual } \\
\text { por fases }\end{array}$ & 26.47 & .000 & .431 & 21.77 & .000 & .383 \\
\hline $\begin{array}{l}\text { Dimensión de Procesos Producción y } \\
\text { Programación }\end{array}$ & 20.63 & .000 & .371 & 12.34 & .001 & .261 \\
\hline
\end{tabular}

En esta dimensión el impacto inmediato muestra un mayor impacto en el conjunto de las tres dimensiones evaluadas (Tabla 5). En todos sus indicadores las diferencias son estadísticamente significativas y relevantes. El indicador integrado de la dimensión 3 que manifiesta un impacto relevante con una diferencia significativa de $\left(\mathrm{F}=20.63, \mathrm{p}<.05, \eta^{2}=.371\right)$.

La pregunta que mejor resultado obtuvo fue la (Pr.16) con un impacto significativo $\left(\mathrm{F}=26.47, \mathrm{p}<.05, \eta^{2}=.431\right)$. Igualmente, los datos son significativos con la pregunta (Pr.15) y (Pr.14). En esta primera pregunta 
con un impacto de $\left(\mathrm{F}=10.49, \mathrm{p}<.05, \eta^{2}=.226\right)$, y en la segunda pregunta, los datos son de un impacto de $\left(\mathrm{F}=9.89, \mathrm{p}<.05, \eta^{2}=.216\right)$.

Dichos resultados pueden considerarse muy exitosos y relacionarse con la sesión Procesos de grabación, donde se realizó una construcción de guiones (literario y técnico) y una grabación siguiendo el guion técnico.

En el impacto diferido se mantienen los buenos resultados de esta dimensión. La diferencia de las medias es estadísticamente significativa, así como el tamaño del efecto apreciable, con un $\left(\mathrm{F}=12.34, \mathrm{p}<.05, \eta^{2}=.261\right)$.

Igualmente, los resultados en la pregunta (Pr.16), son estadísticamente significativos con un impacto significativo de $\left(F=21.77, p<.05, \eta^{2}=.383\right)$. La pregunta (Pr.14) alcanzó un impacto de $\left(\mathrm{F}=8.94, \mathrm{p}<.05, \eta^{2}=.199\right)$. Los cambios producidos en el grupo experimental se han interiorizado y se mantienen a lo largo el tiempo, igualmente que el aprendizaje fue constructivo.

Dimensión de la transformación de la realidad a través de la comunicación

\section{Tabla 6}

Impacto inmediato y diferido Dimensión de la transformación de la realidad a través de la comunicación - Grupos Bogotá

\begin{tabular}{lccccccc}
\hline \multicolumn{1}{c}{ Variables } & \multicolumn{2}{c}{ ANCOVA T2 } & \multicolumn{3}{c}{ ANCOVA T3 } \\
\hline \multicolumn{1}{c}{ Dimensión Transformación realidad } & F & Sig. & Eta $^{2}$ & F & Sig. & Eta $^{2}$ \\
\hline $\begin{array}{l}\text { Pr.3: Si el anuncio influye en la deci- } \\
\text { sión de compra de una persona ¿por qué } \\
\text { crees que lo logra? }\end{array}$ & .01 & .909 & .000 & .29 & .592 & .008 \\
\hline $\begin{array}{l}\text { Pr.4: Cuando las noticias van acompa- } \\
\text { ñadas de imágenes no hay tanto riesgo } \\
\text { de ser manipulado, porque gracias a las } \\
\text { imágenes se puede ver la realidad }\end{array}$ & & & & & & & \\
\hline $\begin{array}{l}\text { Pr.11: Si crees que el anuncio de Coro- } \\
\text { lla no se basa en la razón, ¿cómo piensas }\end{array}$ & .00 & .997 & .000 & .11 & .746 & .003 \\
$\begin{array}{l}\text { que consigue que nos interesemos por el } \\
\text { coche? }\end{array}$ & & & & & & & \\
\hline Pr.19.1: Programación & .01 & .913 & .000 & .96 & .333 & .026 \\
\hline Pr.19.2: Lenguaje & .13 & .723 & .004 & .12 & .736 & .003 \\
\hline Pr.19.3: Publicidad & 4.49 & .041 & .111 & 4.43 & .042 & .110 \\
\hline Pr.19.4: Derechos Humanos & .04 & .838 & .001 & .11 & .738 & .003 \\
\hline Pr.19.5: Información & 1.27 & .267 & .034 & .09 & .770 & .002 \\
\hline $\begin{array}{l}\text { Pr.19: ¿Hay razones para quejarse de } \\
\text { algunas televisiones? }\end{array}$ & .51 & .479 & .014 & .04 & .835 & .001 \\
\hline & & & & & & & \\
\hline
\end{tabular}




\begin{tabular}{lccccccc}
\hline \multicolumn{1}{c}{ Variables } & \multicolumn{3}{c}{ ANCOVA T2 } & \multicolumn{3}{c}{ ANCOVA T3 } \\
\hline \multicolumn{1}{c}{ Dimensión Transformación realidad } & F & Sig. & Eta $^{2}$ & F & Sig. & Eta $^{2}$ \\
\hline $\begin{array}{l}\text { Pr.21: La opción más apropiada para } \\
\text { discutir sobre lo visto en el último capí- } \\
\text { tulo de una serie favorita de televisión }\end{array}$ & 4.71 & .037 & .116 & .13 & .723 & .004 \\
\hline Pr23.1: Trabajo colectivo & .60 & .445 & .018 & .77 & .388 & .023 \\
\hline Pr23.3: Participación ciudadana & 2.80 & .104 & .078 & 2.98 & .093 & .079 \\
\hline Pr23.4: Debate & 2.80 & .104 & .078 & 3.98 & .054 & .102 \\
\hline Pr23: Comentario y/o cotilleo & 2.60 & .117 & .073 & .21 & .653 & .006 \\
\hline
\end{tabular}

En esta dimensión, los resultados significativos del impacto inmediato se reflejan en dos preguntas de dos categorías diferentes. La primera categoría de Competencia comunicativa con la pregunta (Pr.21) con un $\left(\eta^{2}=.116\right)$ y con una significación de $(\mathrm{F}=4.71, \mathrm{p}<.05)$. Dicho resultado podría relacionarse con el consumo televisivo que muestran los adolescentes y el manejo frecuente de apps para mantener conversaciones on-line con sus grupos de amigos. La segunda categoría es Conciencia receptora y crítica que, a través de la pregunta (Pr.19) alcanzó un $\left(\mathrm{F}=4.49, \mathrm{p}<.05, \eta^{2}=.111\right)$ en el ítem referido a la publicidad. Con estos resultados, podría inferirse que el alumnado de este contexto presenta una mayor tendencia a transmitir quejas por la publicidad emitida en algunas de las televisiones, a largo plazo. Los resultados en esta dimensión podrían relacionarse con la sesión de Anuncios y Quejas y Cinco preguntas claves donde los anuncios trabajados en esta sesión contribuyeron a un ambiente de debate entre el alumnado.

Estos resultados, en la Tabla 6, permiten comprobar que el alumnado, tras la intervención, está más familiarizado y es más eficaz para identificar las figuras de Participación ciudadana, Debate y Comentario-cotilleo. Estos conceptos se trabajaron en la sesión Cinco preguntas claves y podría afirmarse que es una de las sesiones que mejores resultados ha obtenido en la intervención.

En el impacto diferido, en esta dimensión, se observan resultados estadísticamente significativos en la categoría de Conciencia receptora y crítica, en la pregunta (Pr.4) con una diferencia de $(\mathrm{F}=11.46, \mathrm{p}<.05$, $\eta^{2}=.241$ ), manteniéndose de este modo este aprendizaje, transcurridos tres meses. Básicamente el alumnado bogotano reconoce que las imágenes son susceptibles de ser manipuladas, que existen técnicas de persuasión y que los mensajes están condicionados por una ideología y unos valores. En la pregunta (Pr.19) de esta misma categoría, los resultados conservaron unas diferencias importantes con un $\left(\mathrm{F}=4.43, \mathrm{p}<.05, \eta^{2}=.110\right)$ en el ítem de la publicidad, manifestando que la actitud crítica frente a la publicidad se mantiene a lo largo del tiempo. 


\section{Tabla 7}

Comparación del Impacto inmediato y diferido entre los contextos en cada dimensión

\section{Preguntas}

\section{Bogotá}

Test 1 Test 2 Test $1 \quad$ Test 2

\section{Lenguaje y Comprensión}

Pr.7: Por qué has elegido esta fotografía

Pr.8: Crea una historia coherente a partir de un conjunto de imágenes

Pr.10: Las tres frases que mejor reflejan los motivos estéticos por los cuales has elegido el anuncio
Pr.12: Selecciona la frase con la que más te identificas

\section{Producción y programación}

Pr.14: Unir las profesiones (audiovisuales) que aparecen en la columna de la izquierda $\mathrm{X}$ con las definiciones de la columna derecha

Pr.15: Señalar a qué proceso de producción corresponde una secuencia, planteada a través de un gráfico

Pr.16: Ordenar las acciones necesarias para la realización de un audiovisual por fases

Dimensión de Procesos Producción y Progra$\mathrm{X}$ mación

\section{Transformación de la realidad}

Pr.4: Cuando las noticias van acompañadas de imágenes no hay tanto riesgo de ser manipulado, porque gracias a las imágenes se puede ver la realidad

\begin{tabular}{lllll}
\hline Pr.19.2: Lenguaje & $\mathrm{X}$ & & & $\mathrm{X}$ \\
\hline Pr.19.3: Publicidad & & & $\mathrm{X}$ \\
\hline $\begin{array}{l}\text { Pr.19: ¿Hay razones para quejarse de algu- } \\
\text { nas televisiones? }\end{array}$ & $\mathrm{X}$ & & & \\
\hline $\begin{array}{l}\text { Pr.21: La opción más apropiada para discu- } \\
\text { tir sobre lo visto en el último capítulo de una } \\
\text { serie favorita de televisión }\end{array}$ & $\mathrm{X}$ & & $\mathrm{X}$ \\
\hline Pr23.4: Debate & & & \\
\hline Pr23.5: Comentario y/o cotilleo & $\mathrm{X}$ & $\mathrm{X}$ & \\
\hline
\end{tabular}


En la Tabla 7 se realiza una comparación, a modo de síntesis, de los resultados significativos del impacto inmediato y diferido que cada contexto logró después de la intervención en cada dimensión.

\section{DISCUSIÓN}

En términos globales, los cambios que se han generado en los diferentes contextos culturales en las áreas de las Competencia Mediática e Informacional, a partir de los resultados obtenidos, cabe destacar los cambios favorables que presenta el contexto español en la dimensión de Lenguaje y comprensión crítica en los resultados del impacto inmediato; y en el contexto colombiano en la dimensión de Procesos de Producción tanto en el impacto inmediato como diferido.

En la dimensión de Lenguaje y comprensión crítica, las categorías de Lectura y argumentación de imágenes, Creación a partir de imágenes y Criterios estéticos y juicios de valor el contexto español obtuvo resultados significativos en el impacto inmediato que se mantuvieron en el impacto diferido, solo en la categoría de Criterios estéticos y juicios de valor.

Los resultados en esta dimensión podrían estar relacionados, en el caso colombiano, con la dificultad que tienen los adolescentes en el desarrollo del pensamiento abstracto y complejo, pensamiento necesario en la lectura y argumentación crítica de textos audiovisuales como en la creación de textos multimedia. Sin embargo, en la adolescencia existe una disposición para expresar y aprender a través de actividades relacionadas con los sentimientos, la identidad y la cultura juvenil, como se percibe en los resultados de la categoría de Criterios estéticos y juicios de valor.

En la dimensión de Procesos de producción y programación, en el contexto colombiano, los resultados fueron estadísticamente significativos tanto en el primero como en el segundo postest.

En el contexto español, en la categoría de Fases de producción, se alcanzaron resultados estadísticamente significativos en el impacto diferido y no en el impacto inmediato. Los buenos resultados en el primer contexto pueden estar relacionados con las actividades prácticas que se trabajaron para desarrollar esta dimensión y, sobre todo, por la implicación del profesorado en la elaboración de guiones y grabaciones con el alumnado, que permitieron que los conocimientos se mantuvieran a largo plazo.

Respecto a la dimensión de Transformación de la realidad a través de la comunicación, el contexto colombiano presentó datos estadísticamente 
significativos en la categoría de Conciencia receptora y crítica en las dos pruebas de impacto. De igual manera, el contexto español, en la categoría de Apropiación tecnológica, obtuvo resultados significativos en ambas pruebas. En la categoría de Competencia comunicativa en ambos contextos los resultados fueron estadísticamente significativos en el primer postest pero no en el segundo postest. Estos resultados pueden compartir el análisis de los resultados obtenidos en la dimensión de Lenguaje y comprensión critica, en la medida que reitera que el uso de la tecnología y el consumo de medios de comunicación e información por sí mismos no generan competencias en la lectura, comprensión, interpretación y creación de productos mediáticos, ni de habilidades de conciencia crítica respecto a la información que se consume. En la misma línea de análisis, los resultados en esta dimensión plantean las dificultades que tienen los adolescentes para la elaboración de trabajos colectivos, de implicación en la participación social y política y en la distribución de información. Sin embargo, el trabajo con anuncios de televisión y las actividades diseñadas en la sesión Cinco preguntas claves podría estimular el desarrollo de estas habilidades de metacognición en estas edades y favorecer el pensamiento abstracto que facilita, no solo el pensamiento crítico, sino también la capacidad de adopción de perspectivas y el trabajo colaborativo.

\section{CONCLUSIONES}

La competencia Mediática e Informacional ha sido señalada como una urgencia educativa por organismos internacionales como la UNESCO (2020) o la Unión Europea (2007). No obstante, es una competencia incluida o transversalizada en el sistema educativo español y colombiano en competencias del ámbito de lo digital y del lenguaje. En el caso de España, esta competencia se incluye en la competencia de comunicación lingüística, tratamiento de la información y competencia digital. En el contexto colombiano, se incorpora dentro de la competencia comunicativa. $\mathrm{Al}$ respecto, Bernabéu et al. (2011) y Cabero y Valencia (2021) plantean que la enseñanza en la Competencia Mediática sigue siendo una propuesta que debe ser asumida por gobiernos y organismos internacionales. Esta falta de entidad de la Competencia Mediática e Informacional dentro del sistema escolar tiene el riesgo de que la educación en medios de comunicación e información se reduzca a unidades didácticas esporádicas que complementan otras asignaturas y que dependen de la voluntad pedagógica del profesorado (Pérez Tornero y Varis, 2012).

La aportación de esta investigación, en lo que refiere a la dimensión de Producción y programación, evidencia la importancia de que el profesorado desarrolle sus competencias digitales para favorecer el aprendizaje 
significativo. Es decir, aprovechar dichas competencias como puente entre la cultura digital de los adolescentes y los conocimientos escolares, en la medida en que son los contenidos prácticos, en los cuales el alumnado de ambos contextos alcanzó resultados considerables.

Este trabajo aporta al conocimiento pedagógico un modelo de intervención para poder trabajar desde la escuela las Competencias Mediáticas e Informacionales desde el marco conceptual de la educomunicación, en un periodo tan vulnerable como es la adolescencia. En el sentido práctico, las dimensiones que requieren un trabajo educativo a largo plazo son las dimensiones de Lenguaje y comprensión y Transformación de la realidad. Y las actividades que mejores resultados han suscitados son las actividades de producción audiovisual y trabajo colaborativo.

El programa de intervención que se presenta en esta investigación, dentro de sus limitaciones contextuales, aporta conocimiento al actual vacío curricular relacionado con la alfabetización mediática. Sin olvidar que en la adolescencia los medios de comunicación son un agente de socialización muy relevante que hoy más que nunca es preciso abordar.

\section{AGRADECIMIENTOS}

Este trabajo fue realizado con la ayuda recibida de la convocatoria de Ayudas a los Grupos de Investigación de la Universidad del País Vasco (UPV/ EHU) (Ref. GIU 20/053) y de la Agencia Estatal de Investigación (MINECOG20/ P61/AEI/ 10.13039/501100011033) y el Programa para el perfeccionamiento de personal investigador doctor del Gobierno Vasco (2018). 


\section{REFERENCIAS BIBLIOGRÁFICAS}

Aguaded, J. I., Vizcaíno, A., \& Sandoval, Y. (2019). Competencia mediática y digital. Del acceso al empoderamiento. Grupo Comunicar.

Bernabéu, N., Esteban, N., Gallego, L., \& Rosales, A. (2011). Alfabetización mediática y competencias básica. Ministerio de Educación.

Buckingham, D. (2005). Educación en medios: Alfabetización, aprendizaje y cultura contemporánea. Paidós.

Cabero, J., \& Valencia, R., (2021). Y el COVID-19 transformó al Sistema Educativo: reflexiones y experiencias por aprender. IJERI: International Journal of Educational Research and Innovation, 15, 218-228. https://doi. org/10.46661/ijeri.5246

Comisión Europea (2006). Recomendación 2006/962/CE del Parlamento Europeo y del Consejo, de 18 de diciembre de 2006, sobre las competencias clave para el aprendizaje permanente. https://bit.ly/3rZ49pU

Cuervo, S., Foronda, A., Rodríguez, A., \& Medrano, C. (2019). Media and information literacy: a measurement instrument for adolescents. Educational Review, 71(6), 395-402.

Cuervo, S., \& Medrano, C. (2013). Alfabetizar en los medios de comunicación: más allá del desarrollo de competencias. Teoría de la Educación. Revista Interuniversitaria, 25(2), 111-131. https://bit.ly/3qPEP4h

Elías, C., \& Catalán-Matamoros, D. (2020). Coronavirus en España: el miedo a las noticias falsas 'oficiales' impulsa WhatsApp y fuentes alternativas. Medios y comunicación, 8(2), 462-466. http://dx.doi. org/10.17645/mac.v8i2.3217
Ferrés, J., \& Piscitelli, A. (2012). Media competence. Articulated proposal of dimensions and indicators. [La competencia mediática: Propuesta articulada de dimensiones e indicadores]. Comunicar, 38, 75-82. https://doi.org/10.3916/C38-2012-02-08

Férres, J., \& Velilla, J. S. (2011). Competencia Mediática: Investigación sobre el grado de competencia de la ciudadanía en la Comunidad Autónoma de La Rioja. Grupo Comunicar.

Garbe, A., Ogurlu, U., Logan, N., \& Cook, P. (2020). Parents' experiences with remote education during COVID19 school closures. American Journal of Qualitative Research, 4(3), 45-65. https://doi.org/10.29333/ajqr/8471

Harshman, J. (2017). Developing a globally minded, critical media literacy. Journal of Social Studies Education Research, 8(1), 69-92. https://bit.ly/3rYIi1F

Hoechsmann, M., \& Poyntz, S. R. (2012). Media literacies: A critical introduction. John Wiley \& Sons.

Lau, J. (2013). Conceptual Relationship of Information Literacy and Media Literacy. In A. Lee, J. Lau, T. Carbo, \& N. Gendina, Conceptual Relationship of Information Literacy and Media Literacy. (pp. 76-91). UNESCO.

Lee, A., Lau, J., Carbo, T., y Gendina, N. (2013). Conceptual relationship of information literacy and media literacy in knowledge societies. UNESCO.

LOE (2006). Ley Orgánica de Educación 2/2006. BOE 4 de mayo.

LOMCE (2013). Ley Orgánica para la mejora de la calidad educativa. 8/2013. BOE 9 de diciembre. 
LOMLOE (2020). Ley Orgánica de Educación. 3/2020. BOE 30 de diciembre.

Mediasmarts (2014). Digital Literacy Fundamentals. https://bit.ly/2NopGJl

Medrano, C., Cuervo, S., \& Martínez de Morentin, J. I. (2019) Programa para favorecer las competencias mediáticas e informacionales de los adolescentes. Pirámide.

MEN (2006). Estándares básicos de competencias en Lenguaje, Matemáticas, Ciencias y Ciudadanía. Guía sobre lo que los estudiantes deben saber y saber hacer con lo que aprenden. Ministerio de Educación Nacional. https://bit.ly/3bTOTF9

Murillo, F. J., y Duk, C. (2020). El covid-19 y las brechas educativas. Revista latinoamericana de educación inclusiva, 14(1), 11-13. https://bit. ly/3gSFxMl

Pérez Tornero, J. M., \& Varis, T. (2012). Alfabetización mediática y nuevo humanismo (Vol. 2). UOC.

Redecker, C. (2020) Marco Europeo para la Competencia Digital de los Educadores: DigCompEdu. Universia y Ministerio de Educación y Formación Profesional de España.

UNESCO (1982). Declaración de Grünwald sobre la Educación relativa a los Medios de Comunicación. https:// bit.ly/3rWfLdc
UNESCO (2011). Declaración de Praga sobre alfabetización mediática. https:// bit.ly/3vwzrXa

UNESCO (2014) Declaración de París sobre la alfabetización mediática. Foro Europeo de Medios de Comunicación e Información de la Alfabetización. https://bit.ly/3lpCzQ3

UNESCO (2020). La Declaración de Seúl sobre la Alfabetización mediática e informacional Para Todos y Por Todos. https://bit.ly/2OY1LB8

Unión Europea (2007) Directiva 2007/65/CE sobre la coordinación de determinadas disposiciones legales, reglamentarias y administrativas de los Estados miembros relativas al ejercicio de la radiodifusión televisiva actividades. https://bit.ly/3rWNwLg

Unión Europea (2018). Recomendación relativa a las competencias clave para el aprendizaje permanente (2018/C 189/01). https://bit.ly/3yMhxBt

Villa, A., \& Poblete, M. (2007). Aprendizaje basado en competencias. Una propuesta para la evaluación de las competencias genéricas. Mensajero/ ICE Universidad de Deusto.

Wilson C, Grizzle A, Tuazon R, Akyempong K., \& Cheung C.K. (2011). Alfabetización Mediática e Informacional. Curriculum para profesores. UNESCO. https://bit. ly/3eJK0QN 


\section{PERFIL ACADÉMICO Y PROFESIONAL DE LOS AUTORES}

Sandra Liliana Cuervo Sánchez. ORCID: http://orcid.org/0000-0003-35408615

Doctora por la Universidad del País Vasco (UPV/EHU) e investigadora permanente de la Cátedra UNESCO de Comunicación y Valores Educativos con una beca de investigación post-doctoral. Sus líneas de investigación son la competencia mediática e informacional; la alfabetización mediática; la desinformación y la infopandemia y los riesgos en las redes sociales. E-mail: sandraliliana.cuervo@ehu.es

Juan-Ignacio Martínez-de-Morentin. ORCID: http://orcid.org/0000-00029268-6470

Profesor pleno (categoría equivalente a la de profesor catedrático en el sistema de contratación universitario vasco) en el Departamento de Psicología Evolutiva y de la Educación, en la Facultad de Psicología de la Universidad del País Vasco (UPV/EHU). Responsable de la Cátedra UNESCO de Comunicación y Valores Educativos, sus líneas de investigación se centran en los valores educativos y la alfabetización mediática, la anomia digital, la percepción del riesgo en las redes sociales y las acciones de riesgo en Internet. Email: juanignacio. demorentin@ehu.es

Concepción Medrano Samaniego. ORCID: http://orcid.org/0000-0001-7980$843 \mathrm{X}$

Colaboradora Honorífica para la Investigación de la Universidad del País Vasco (UPV/EHU) e investigadora permanente de la Cátedra UNESCO de Comunicación y Valores Educativos en dicha Universidad. Sus líneas de investigación se centran en el estudio de las relaciones entre los medios de comunicación, como agentes de socialización en la infancia y en la juventud, y la construcción de la identidad y los valores, con una proyección respecto a la alfabetización mediática e informacional. Asimismo, en el análisis del uso y los riesgos de Internet, tanto en menores como en adultos. E-mail: mariaconcepcion. medrano@ehu.es

Fecha Recepción del Artículo: 26. Marzo. 2021

Fecha Modificación del Artículo: 03. Junio. 2021

Fecha Aceptación del Artículo: 03. Julio. 2021

Fecha Revisión para Publicación: 13. Septiembre. 2021 